\title{
GAMMA SHIELDING EXPERIMENT SIMULATION UTILIZING MCNPX CODE
}

\author{
Shafiqul Islam Faisal ${ }^{1 *}$ and Abi Muttaquin Bin Jalal Bayar ${ }^{2}$ \\ ${ }^{1}$ Bangladesh Atomic Energy Regulatory Authority, E-12/A, Agargaon, Dhaka-1207, Bangladesh \\ ${ }^{2}$ Malaysian Nuclear Agency, Bangi, 43000 Kajang, Selangor, Malaysia
}

Received: 19 January 2021

Accepted: 11 May 2021

\begin{abstract}
Experimental investigation requires materials, radiation sources, and test arrangements with a high monetary financial plan. Furthermore, radiation exposure involves people during the experiment. On the contrary, the simulation technique for examining radiation interactions is radio-logically safer, less timeconsuming, cost-effective, and applicable for all desired radiation sources. Through $48.86 \mathrm{mCi} 662 \mathrm{keV}$ Caesium-137 gamma-ray source; shielding experiment as well as simulation of it with MCNPX were performed for three shielding materials Lead, Copper, and Aluminum. These materials were placed in front of the gamma source and the emergent radiation was counted in a Geiger-Muller detector to understand the attenuation quality of these materials to each other. These courses of action were simulated utilizing the MCNPX code version 2.7.0 and the results likewise gave and looked at that of the experiment. There are huge similarities of shielding behavior between MCNPX simulation and experiments for the three absorbing materials. The modeled geometry of this MCNPX simulation could be used for future approaches of new designs and structures of radiation shielding, especially where no analogous experimental data exist
\end{abstract}

\section{Keywords: Gamma Shielding, MCNPX Code, Simulation}

\section{INTRODUCTION}

The exploit of distinctive gamma-ray sources have been enforced in various fields in medicine, industries, agriculture, nuclear power plants, etc. Though using gamma-rays have preferences in several regions, destructive impacts on human wellbeing cannot be overlooked. Gamma-rays may be the cause of different diseases (such as cancer, radiation sickness, mutations, etc.) due to their high energy and penetrability. Radiation shielding stays a significant part of radiation science. Shielding is extremely critical in industries, radioisotopes production, medical practices, and particle accelerator facilities. Thus, it is obligatory to use and develop materials in radiation shielding applications. The shielding materials choice is dependent upon the necessity for an exposure rate reduction, type of source, space constraints, and cost-effective analysis.

Applying The Monte Carlo technique is perhaps the best response for the study of various materials behavior where experimental replication of analysis is very convoluted. Monte Carlo N-Particle (MCNP) code is especially helpful for complex issues that can't prove by the deterministic method of computer codes. MCNP is a code used for photon, neutron, and electron or coupled neutron/photon/electron transport. Utilization of MCNP code is in radiation shielding, radiation protection and dosimetry, radiography, medical physics, nuclear oil well logging, detector design and analysis, accelerator target design, fission, and fusion reactor design, reactor criticality safety, and decommissioning (Judith F. Briesmeister, 2000). MCNP is a code undergoing continuous development at Los Alamos National Laboratory (LANL) and has periodic new releases. Monte Carlo N-Particle eXtended (MCNPX) was also developed at LANL, is capable of simulating particle interactions of 34 different types of particles (nucleons and ions) and 2000+ heavy ions at nearly all energies, including those done by MCNP (Metropolis N., 1987). Monte Carlo simulations are applied in several territories of physics and chemistry including realistic simulations of modeled physical systems but are of specific significance in the demonstrating of radiation transport. The Monte Carlo method is the stochastic based complex programming code that can give the user desired outcomes of fluency, energy fluency, absorbed dose, equivalent dose, effective dose, number of photons, and so forth.

This work aims to simulate the gamma shielding experiment in MCNPX code version 2.7.0. The major goal of the current study is to validate the shielding estimations in MCNPX code for experimental works in the point sources of ${ }^{137} \mathrm{Cs}$ gamma rays. For this purpose, the effective shielding factor half-value layer (HVL) is figured for the three absorbers such as Lead, Copper, and Aluminum at gamma photon of energy $662 \mathrm{keV}$ from 
experimental data as well as utilizing the MCNPX code. Simulation results also assimilate with the theoretical values.

\section{MATERIALS AND METHOD}

\subsection{Laboratory Experiment of Gamma-ray Shielding for HVL Measurement}

Figure-1 shows the laboratory experimental setup for HVL measurement. The gamma source activity of 48.86 $\mathrm{mCi}$ was used during the experiment. A Geiger Muller (GM) detector was used to detect the gamma rays at 50 $\mathrm{cm}$ distance (from the source to the outer surface of the detector). Different thickness Lead, Copper, and Aluminum absorber sheets shows that Figure- 2 were placed one by one in front of the source container stand in between the source and the detector. Each sheet of $\mathrm{Pb} \& \mathrm{Cu}$ absorber thickness is $0.1 \mathrm{~cm}$ and $\mathrm{Al}$ is $0.5 \mathrm{~cm}$. Absorber sheets were added to increase the thickness and the corresponding variation of gamma-ray counts was recorded from GM detector.

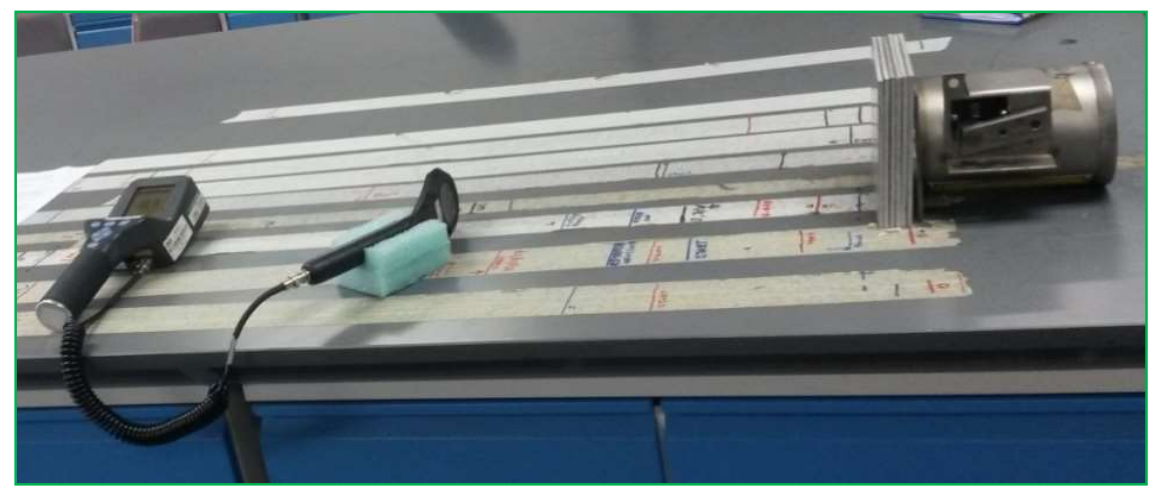

Figure 1: Laboratory Experiment Set up

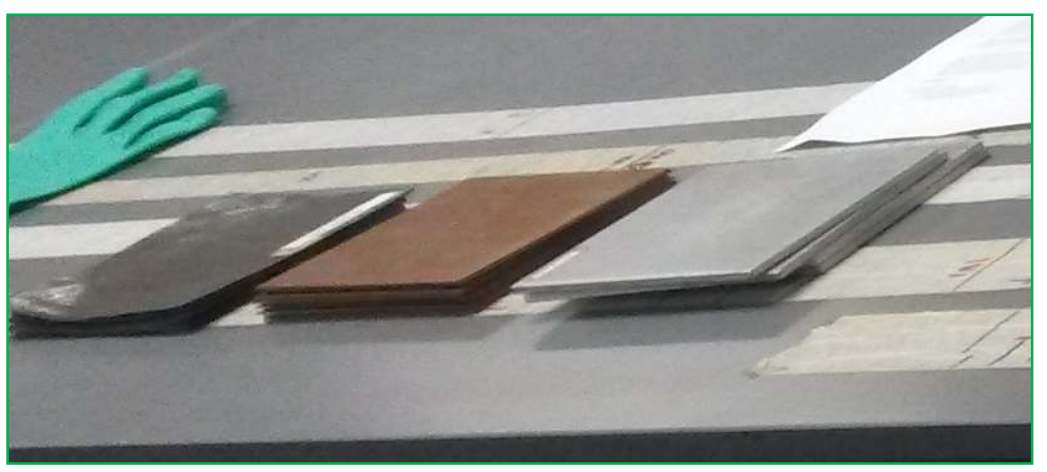

Figure-2: Various thickness of shielding material (Lead, Copper \& Aluminum)

While a gamma-ray traverses an absorber, the intensity of the beam will be lessened by Beer-Lambert's law. The alteration of intensity for a photon in good geometry is typically expressed mathematically as a decreasing function with the thickness of the absorber. The number or intensity I(x) of photon penetrating an absorber of thickness $\mathrm{x}, \mathrm{I}_{0}$ is the gamma-ray intensity at zero absorber thickness

$$
I=I_{0} e^{-\mu x}
$$

$\mu$ is the constant of proportionality which is the total attenuation coefficient of the medium for the photon of interest. The linear attenuation coefficient varies with photon energy, type of material, and physical density of the material and is the probability of interaction per unit distance in an absorbing medium.

The thickness of any given material where $50 \%$ of the incident photon has been attenuated is known as the halfvalue layer (HVL). The HVL is in units of distance $(\mathrm{mm}$ or $\mathrm{cm})$. If the incident intensity of 1 and transmitted intensity 0.5 is plugged into the equation (1) 


\section{$0.5=1.0 e^{-\mu x}$}

The values of HVL thicknesses are, $H V L=x_{1 / 2}=\ln 2 / \mu$

The HVL is inversely proportional to the attenuation coefficient. Like the attenuation coefficient, it is photon energy-dependent. Increasing the penetrating energy of a stream of photons will increase the material's HVL.

\subsection{MCNPX Simulation Basis}

The strategy of MCNPX involves the modeling of a physical system by statistically simulating individual physical events of large numbers. The individual probabilistic events that form a process are simulated sequentially. The probability distributions governing these events are statistically sampled to describe the total phenomenon. In general, the simulation is performed on a digital computer because the number of trials necessary to adequately describe the phenomenon is usually quite large. Depending on the situation defined, it runs millions of historical data to provide the exact solution wanted. The number of minutes or hours it will run or the number of particles it will follow can also be defined. At the end of the simulation, information about the properties of the system is gathered. The statistical sampling process is based on the selection of random numbers-analogous to throwing dice in a gambling casino-hence the name "Monte Carlo" (X-5 Monte Carlo Team, 2005). In particle transport, the Monte Carlo technique is pre-eminently realistic (a numerical experiment) consists of actually following each of many particles from a source throughout its life to its death in some terminal category (absorption, escape, etc.). Probability distributions are randomly sampled using transport data to decide the outcome at each step of its life (X-5 Monte Carlo Team, 2005). MCNPX uses continuous-energy nuclear and atomic data libraries (Boston Andrew, 2000). For neutrons, all reactions are given in a particular cross-section evaluation (e.g. ENDF/B-VI) are accounted for. For photons, the code takes account of incoherent and coherent scattering, the possibility of fluorescent emission after photo-electric absorption, absorption in pair production with local emission of annihilation radiation, and bremsstrahlung.

In MCNPX, the user makes an input file that is in this way read by MCNPX. The maximum line length in the MCNPX input file is 80 columns and it has a fixed structure that adheres to give a reliable output. Every input file defines a series of 'cards' or commands containing parameters (geometry of the problem, source definition, materials definition, and tallies to output results). Cell cards are utilized to characterize the shape and material substance of physical space. Cells are the fundamental units of MCNPX. It uses Cartesian axes of X, Y, Z and it can be set randomly. Any cell is characterized by encompassing surfaces and used to intersections, unions, and complements of the regions bounded by the surfaces of the problem objects. The Surface card characterizes the limits in space used to make cells (spheres, cylinders, planes, and so forth.). Surfaces are in the analytic surface equations or macro bodies. Numerous surfaces are joined to frame cells which are characterized and marked. All surfaces are characterized in the Cartesian coordinate framework. These surfaces together produce a geometry that represents the actual problem which is desired to be understood by MCNPX. Source definitions card contains the source and sort of radiation particles for the MCNPX problem are determined by the SDEF command, which conveys a few parameters like - Pos: for $\mathrm{x}, \mathrm{y}, \mathrm{z}$ position of problem radioactive source. - Erg: defines the energy of the emitted particle, with energy distribution d1. Tallies card (F's cards) is used to specify the type of information we have to obtain from the MCNP simulation, like particle current, flux, energy, etc. Material specification card includes the elements of defining a unique material number, element composition or isotopic composition, and cross-section compilations that are used during the simulation.

The key thoughts of the MCNPX technique are precision, exactness, central limit hypothesis, count mean (error), and count variances. Each MCNPX output incorporates the evaluated relative error. The estimated relative error gives a confidence interval about the evaluated quantity. An overall error of under 0.1 is a decent sign that the tally is reliable; a relative error between 0.1 and 0.2 demonstrates that the true outcome is inside a significant degree, yet a relative error of 0.2 to 0.5 isn't excessively dependable and the simulation ought to be run with a larger number of particle histories. If relative error is more significant than 0.5 , at that point results are meaningless.

\subsection{Geometry of Gamma Experiment for the MCNPX Code}

To run MCNPX first the geometry of the Laboratory experiment has been defined accurately is appeared in Figure-3. The code treats an arbitrary three-dimensional configuration of materials in geometrical cells bounded by first and second-degree surfaces (X-5 Monte Carlo Team, 2005). Generally, a world is additionally defined. A world in the region of interest and the particle will be followed wherever it goes within this world. Inside the world, it is conceivable to find out every bit of information about particle-like velocity, energy, acceleration, and so on. The simulated particle won't be followed and considered assimilated outside the world. 
The allocation of the Cartesian axes $\mathrm{X}, \mathrm{Y}$, and $\mathrm{Z}$ is arbitrary. The source is considered at $(0,0,8.5)$ Cartesian coordinate in the MCNPX geometry assumes the source is in the center of the $17 \mathrm{~cm}$ height shielded encapsulate. The Cs-137 source is encased with shielded stainless steel (SS)-316L container of $17 \mathrm{~cm}$ height and a $5.75 \mathrm{~cm}$ radius. In front of the cylindrical shape shielded container, there are welded Aluminum and SS-316L fixed rectangular barrier sheets of thickness $1 \mathrm{~mm}$ and $2 \mathrm{~mm}$ respectively. Both $\mathrm{Al}$ and SS-316L are in length $12.5 \mathrm{~cm}$, width $12.5 \mathrm{~cm}$. Since the actual source dimension couldn't gather from any source information detail or some other way, the source dimension was back-calculated from the specific activity of Cs-137 source and the volume of the gamma source is found as $5.96 \mathrm{e}-4 \mathrm{~cm}^{-3}$. From that source volume, the radius of the source is determined as $0.044 \mathrm{~cm}$ considering the length of the source is $0.1 \mathrm{~cm}$. Assumed the source is to be a cylindrical shape. A tube-shaped air collimator is appended before the Cs-137 source. The photon emergent and reaching the detector is to be air medium while outside the framework is thought to be void. $\mathrm{Pb}, \mathrm{Cu} \& \mathrm{Al}$ absorber sheet surface area is $\left(15^{*} 15\right) \mathrm{cm}^{2}$ and the GM detector probe circular surface area is $15 \mathrm{~cm}^{2}(2.185 \mathrm{~cm}$ radius $)$

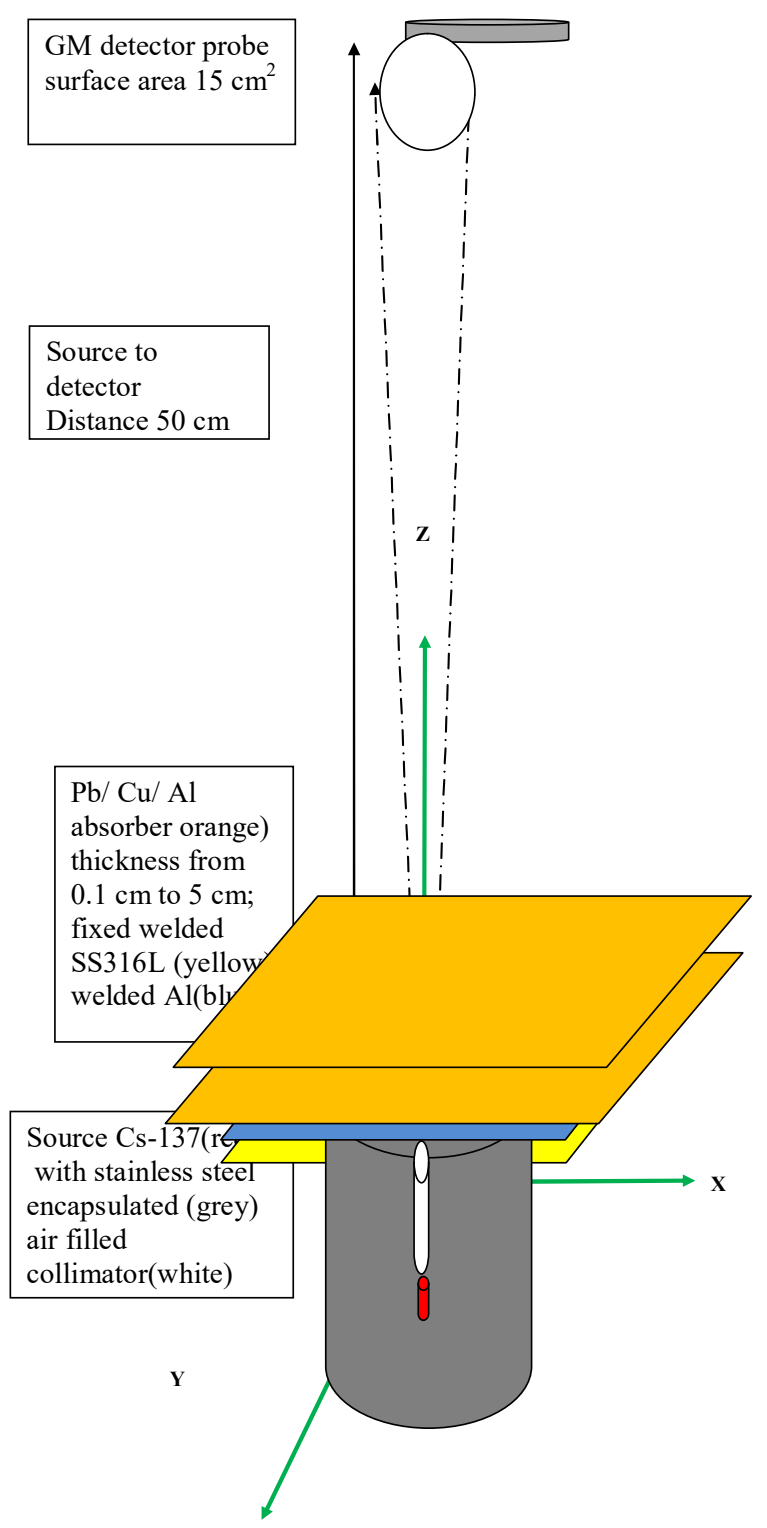

Figure 3: Schematic diagram for MCNPX problem 


\section{RESULTS AND DISCUSSIONS}

Both the experimental results and the MCNPX outputs were standardized by dividing the counts by the counts at zero thickness. This was done so that the two outputs can be compared easily.

\subsection{Laboratory Experiment Results}

The experimental results for Lead, Copper and Aluminum absorbers photon intensity with increasing absorber thickness are shown in Figure-4. The results show that the peak photon count which is always highest at zero absorbers thickness, i.e. when no absorbing material was inserted $\left(\mathrm{I}_{0}\right)$ continues to decrease in the count with increasing absorber thickness in each of the three absorber materials. The graph also shows an exponential decrease in normalized photon count as against increasing absorber thickness.

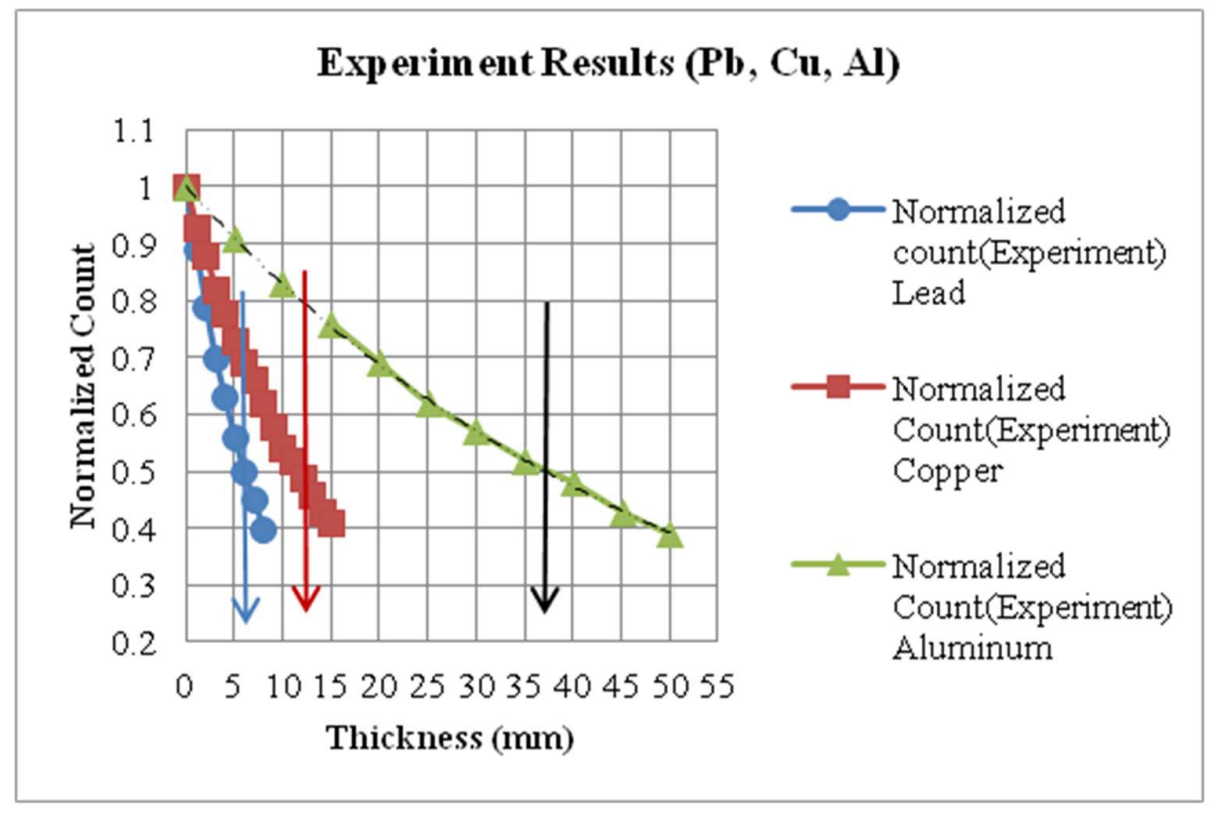

Figure 4: Exponential decrease of gamma photon normalized count against $\mathrm{Pb}, \mathrm{Cu}$, and $\mathrm{Al}$ thickness

\subsection{Laboratory Experiment Simulation in MCNPX Code And Input File}

The MCNPX code deciphers the user-defined input file to decide the problem geometry, source, energy, location, and physics parameters. MCNPX input file is compiled by a simple "notepad++" of operating windows. The code is written on the notepad using the specific syntax of MCNPX and then runs on the Visual Editor (VisEd) of MCNPX by transferring it into the MCNPX input file option. There are thirty-four (34) input files produced in this simulation project for three absorbers $(\mathrm{Pb}, \mathrm{Cu}$, and $\mathrm{Al}$ ) with various thicknesses, ranging from $0.1 \mathrm{~cm}$ to $5.0 \mathrm{~cm}$ including one input file for no absorbing material. Before every running the input file to the MCNPX, it has been checked by VisEd. Consequently, the same number of output files has been generated by MCNPX.

The simulation is executed in the command line prompt. The simulation has been performed using the Intel ${ }^{\circledR}$ Core TM i3 CPU $2.10 \mathrm{GHz}$ computer hardware. The number of photons of the desired energy passing through the GM detector is calculated with the help of the tally $\mathrm{fl}$ and the number of particle histories $10^{8}$ has also been specified in the input files. For all the simulations in MCNPX, the cross-section is chosen from appropriate libraries, considering the nature of the problem being solved and the data available to the user. In this simulation, the cross-section photon transport data library is selected as Mcnplib04 and it was officially released in 2002, maintained by X-Division.In the Input file as shown at right side of Figure 5, the .04p designation identifies the Mcnplib04 cross-section library for incident photon energies from- $1 \mathrm{keV}$ to $1 \mathrm{GeV}$. These crosssections used in the input file are resultant from the Evaluated Nuclear Data File (ENDF)/B-VI.8 data library that was derived from the Evaluated Photon Data Library (EPDL)- 97. A comprehensive list of continuous energy photo atomic cross-section compilations are provided in Table G.4 of Appendix G in the MCNPX user's manual (Denise B. Pelowitz, editor, 2011). The elemental atomic fraction for compounds or mixtures is 
specified by a mass fraction that has been taken from the PNNL material composition data manual. (McConn R. J. Jr, et al, 2011).

Visualization of input file geometry (cells and surfaces) in 2D for the MCNPX simulation of the experimental setup is shown in Figure-5. The zoom view (closer look) of the cell label and surface label of the MCNPX geometry configuration is in Figure-6. In the geometry, the color labeling is as Green- Air Body (cell 90) and air collimator (cell-3) detector (cell 7); Dark Orange- stainless steel 316L (cell 2) and fixed welded SS 316L (cell 4); Dark Red- fixed welded Al barrier; Light Blue- Lead absorber (cell 5); Red spot- Cs-137(cell 1) not shown in the Figure can visible in more zoom. Figure-7 presents the 3D views of experimental arrangement; verification of source through particle tracking (source generation points, sites of collisions, surface crossing) in VisEd.

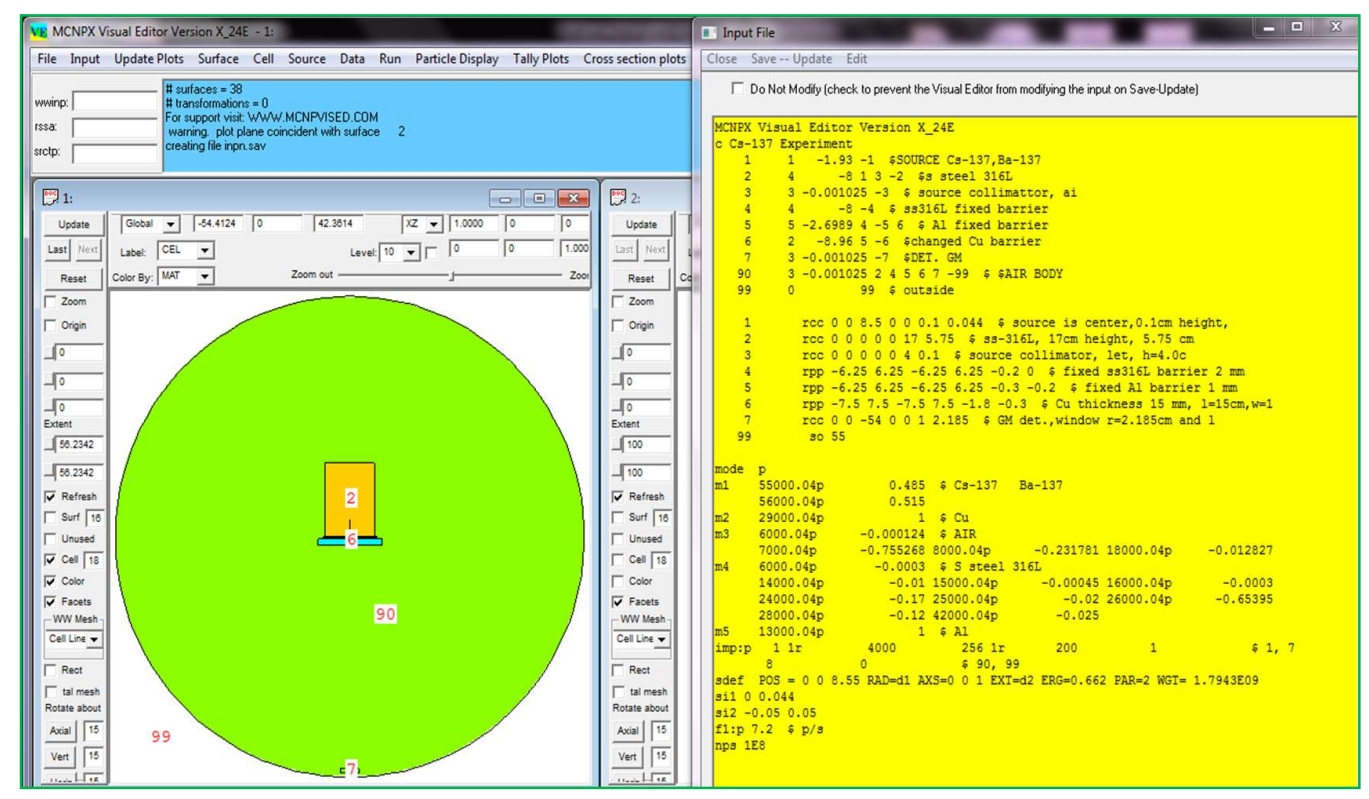

Figure 5: Experimental setup constructed in MCNPX plot with the Cell label in VisEd
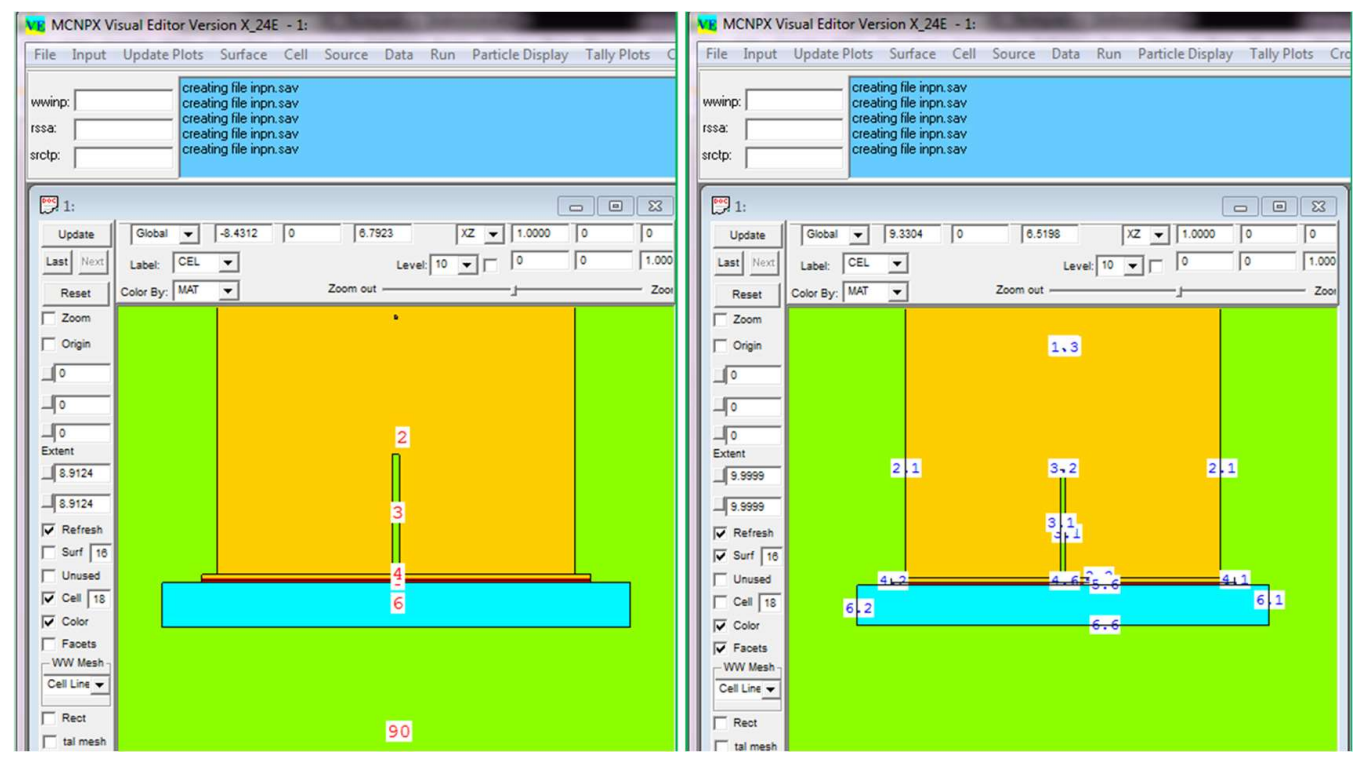

Figure 6: Zoom view of Cell label (left) and Surface label (right) in VisEd 


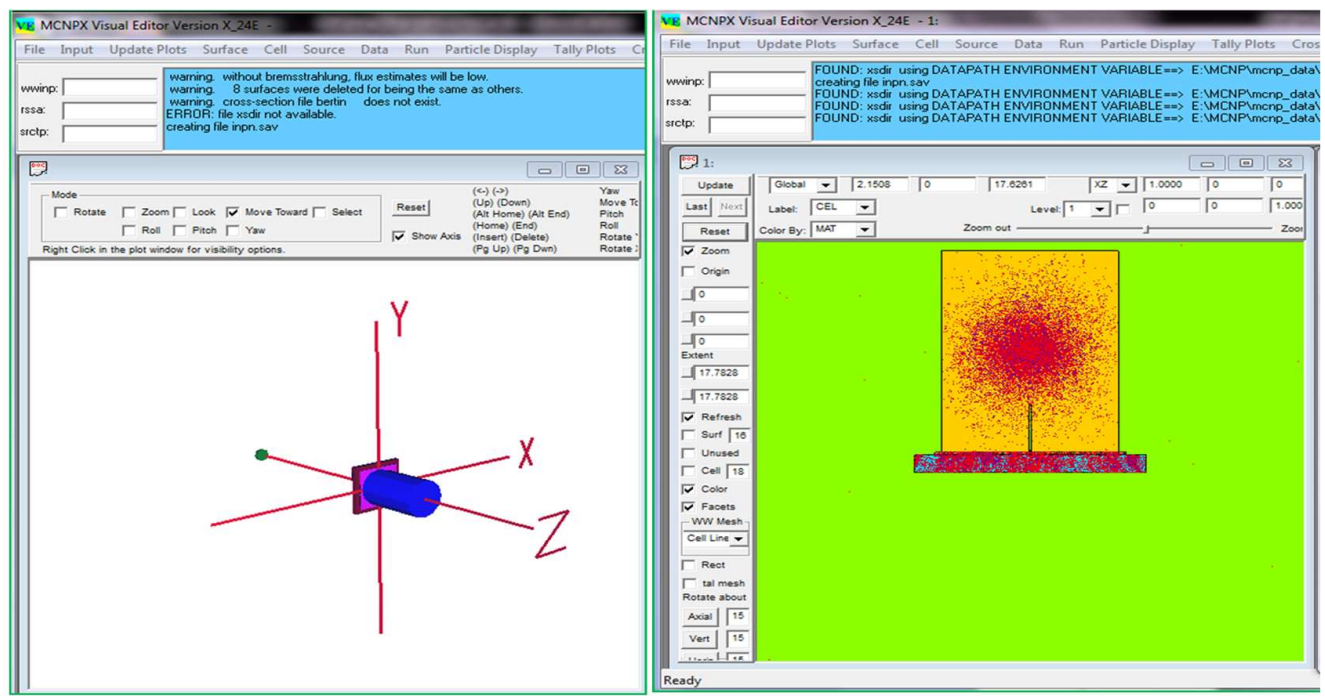

Figure 7: 3D display of the experimental setup (left) and Particle tracking (right) in MCNPX using VisEd

\subsection{MCNPX Simulation Output Result}

MCNPX initiates an output file, which includes a copy of the input file, any flags or warnings the code encounters, and initial statistics about the simulation. When all particle histories finish running, MCNPX runs statistical checks for each tally. Then, MCNPX writes, the final tally results with their corresponding relative errors to the output file. After the tally results, the important parts are the 10 statistical checks which the run should pass for reliable results. The statistical checks for each tally give the user a level of confidence in the result depending on how many checks the tally has passed. A tally is considered to be converged with high confidence only when it passes all ten statistical checks (Denise B. Pelowitz, editor, 2011). These checks include mean behavior; relative error, $\mathrm{R}$ (value, decrease, and decrease rate); the variance of variance, VOV (value, decrease, and decrease rate); the figure of merit, FOM (value, behavior); probability density functional (PDF) slope (X-5 Monte Carlo Team, 2005).

The result of the simulation work for three absorber materials with different thicknesses is presented in Tables 1-3 and Figure 8. For the three materials, normalized MCNPX values vary inversely proportional to the absorber thickness, in an almost exponential form. The overall relative errors of MCNPX outcomes can be declared as "reliable" as the error is less than 0.03 for all thickness and absorber materials.

Table 1: Photon intensity against absorber's $(\mathrm{Pb})$ thickness

\begin{tabular}{cccc}
\hline $\begin{array}{c}\text { Thickness } \\
(\mathrm{mm})\end{array}$ & $\begin{array}{c}\text { MCNPX Results } \\
\text { (Particles } \\
\text { reached to the } \\
\text { detector) }\end{array}$ & $\begin{array}{c}\text { Normalized } \\
\text { count (ratio } \\
\text { relative to zero } \\
\text { thickness) }\end{array}$ & $\begin{array}{c}\text { Relative } \\
\text { error } \\
\text { (MCNPX) }\end{array}$ \\
\hline 0 & $2.87 \mathrm{E}+04$ & 1 & 0.0246 \\
1 & $2.36 \mathrm{E}+04$ & 0.82 & 0.0247 \\
2 & $1.96 \mathrm{E}+04$ & 0.68 & 0.0255 \\
3 & $1.69 \mathrm{E}+04$ & 0.59 & 0.0261 \\
4 & $1.42 \mathrm{E}+04$ & 0.5 & 0.0265 \\
5 & $1.25 \mathrm{E}+04$ & 0.44 & 0.0273 \\
6 & $1.11 \mathrm{E}+04$ & 0.39 & 0.0274 \\
7 & $9.61 \mathrm{E}+03$ & 0.33 & 0.0278 \\
\hline 8 & $8.44 \mathrm{E}+03$ & 0.29 & 0.0286 \\
\hline
\end{tabular}

Table 2: Photon intensity against absorber's $(\mathrm{Cu})$ thickness

\begin{tabular}{cccc}
\hline $\begin{array}{c}\text { Thickness } \\
(\mathrm{mm})\end{array}$ & $\begin{array}{c}\text { MCNPX Results } \\
\text { (Particles } \\
\text { reached to the } \\
\text { detector) }\end{array}$ & $\begin{array}{c}\text { Normalized } \\
\text { count (ratio } \\
\text { relative to zero } \\
\text { thickness) }\end{array}$ & $\begin{array}{c}\text { Relative } \\
\text { error } \\
\text { (MCNPX) }\end{array}$ \\
\hline 0 & $2.87 \mathrm{E}+04$ & 1 & 0.0246 \\
1 & $2.36 \mathrm{E}+04$ & 0.82 & 0.0247 \\
2 & $1.96 \mathrm{E}+04$ & 0.68 & 0.0255 \\
3 & $1.69 \mathrm{E}+04$ & 0.59 & 0.0261 \\
4 & $1.42 \mathrm{E}+04$ & 0.5 & 0.0265 \\
5 & $1.25 \mathrm{E}+04$ & 0.44 & 0.0273 \\
6 & $1.11 \mathrm{E}+04$ & 0.39 & 0.0274 \\
7 & $9.61 \mathrm{E}+03$ & 0.33 & 0.0278 \\
\hline 8 & $8.44 \mathrm{E}+03$ & 0.29 & 0.0286 \\
\hline
\end{tabular}


Table 3: Photon intensity against absorber's (Al) thickness

\begin{tabular}{|c|c|c|c|}
\hline $\begin{array}{c}\text { Thickness } \\
\text { (mm) }\end{array}$ & $\begin{array}{c}\text { MCNPX Results } \\
\text { (Particles } \\
\text { reached to the } \\
\text { detector) }\end{array}$ & $\begin{array}{c}\text { Normalized } \\
\text { Count(ratio } \\
\text { relative to zero } \\
\text { thickness) } \\
\end{array}$ & $\begin{array}{c}\begin{array}{c}\text { Relative } \\
\text { error }\end{array} \\
\text { (MCNPX) }\end{array}$ \\
\hline 0 & $2.87 \mathrm{E}+04$ & 1 & 0.0246 \\
\hline 1 & $2.75 \mathrm{E}+04$ & 0.96 & 0.0239 \\
\hline 2 & $2.59 \mathrm{E}+04$ & 0.9 & 0.0236 \\
\hline 3 & $2.45 \mathrm{E}+04$ & 0.85 & 0.023 \\
\hline 4 & $2.33 \mathrm{E}+04$ & 0.81 & 0.0226 \\
\hline 5 & $2.19 \mathrm{E}+04$ & 0.76 & 0.0224 \\
\hline 6 & $2.12 \mathrm{E}+04$ & 0.74 & 0.0223 \\
\hline 7 & $2.01 \mathrm{E}+04$ & 0.7 & 0.022 \\
\hline 8 & $1.89 \mathrm{E}+04$ & 0.66 & 0.0219 \\
\hline 9 & $1.80 \mathrm{E}+04$ & 0.63 & 0.0216 \\
\hline 10 & $1.68 \mathrm{E}+04$ & 0.59 & 0.0214 \\
\hline 11 & $1.59 \mathrm{E}+04$ & 0.55 & 0.0216 \\
\hline 12 & $1.49 \mathrm{E}+04$ & 0.52 & 0.0214 \\
\hline 13 & $1.44 \mathrm{E}+04$ & 0.5 & 0.0215 \\
\hline 14 & $1.37 \mathrm{E}+04$ & 0.48 & 0.0211 \\
\hline 15 & $1.29 \mathrm{E}+04$ & 0.45 & 0.0215 \\
\hline
\end{tabular}

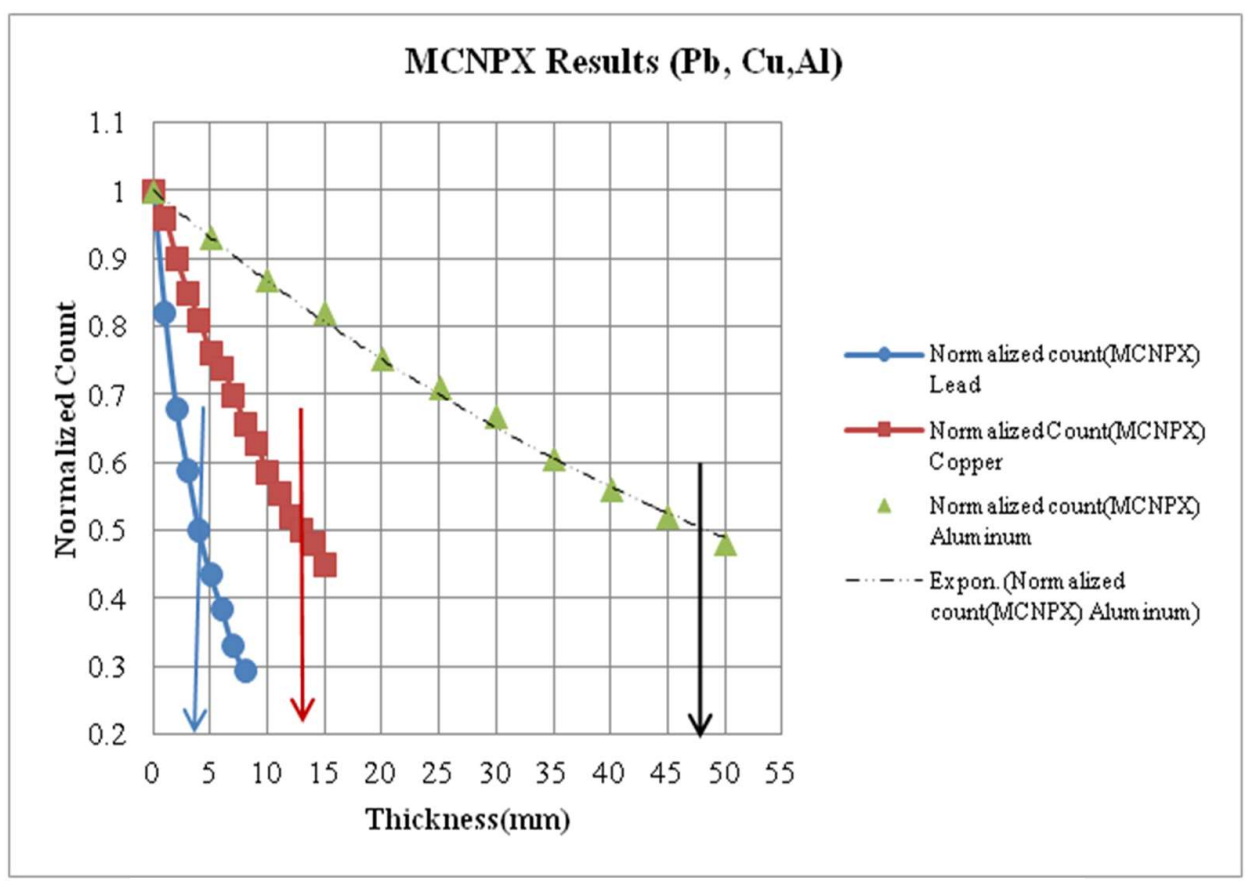

Figure 8: $\mathrm{MCNPX}$ Results for three absorbers $(\mathrm{Pb}, \mathrm{Cu}, \mathrm{Al})$

Figure- 8 shows the comparative MCNPX Results for three absorbers $(\mathrm{Pb}, \mathrm{Cu}, \mathrm{Al})$. The diagrams of these three absorbing materials look fundamentally the same manners and significant distinction among these three materials, particularly Lead. While just $0.44 \mathrm{~cm}$ thickness of Lead is required to decrease the gamma photon intensity or photon count to half of its zero thickness value, about $1.3 \mathrm{~cm}$ of Copper and $4.7 \mathrm{~cm}$ of Aluminum are required to accomplish a similar work that Lead has done in MCNPX simulations. 


\subsection{Comparison of Experimental and MCNPX Simulation Results Along with Theoretical values}

For comparison of the simulation and the experimental results, two sets of plots for each absorber material (Lead, Copper, and Aluminum) present with theoretical value in Figures (9-11).

a) Linear Attenuation Coefficients of Lead at $662 \mathrm{keV}$ energy $(\mu)=1.29 \mathrm{~cm}^{-1}$ (Etherington, H, 1958);

$\mathrm{HVL}=0.693 / \mu=0.693 / 1.29 \mathrm{~cm}^{-1}=0.537 \mathrm{~cm}=5.37 \mathrm{~mm}$

b) Linear Attenuation Coefficients of Copper at $662 \mathrm{KeV}$ energy $(\mu)=0.67 \mathrm{~cm}^{-1}$ (Etherington, $\mathrm{H}, 1958$ );

$\mathrm{HVL}=0.693 / \mu=0.693 / 0.67 \mathrm{~cm}^{-1}=1.03 \mathrm{~cm}=10.3 \mathrm{~mm}$

(c) Linear Attenuation Coefficients of Aluminum at $662 \mathrm{KeV}$ energy $(\mu)=0.21 \mathrm{~cm}^{-1}$ (Etherington, $\mathrm{H}, 1958$ );

$\mathrm{HVL}=0.693 / \mu=0.693 / 0.21 \mathrm{~cm}^{-1}=3.3 \mathrm{~cm}=33 \mathrm{~mm}$

It observes that each pair of the three graphs has similar behavior, in that the photon intensity is inversely proportional to the absorber thickness, in an almost exponential form. As the absorber's thickness increases, the photon intensity decreases; conversely, as reduce the absorber's thickness, the photon intensity increases. This is conceivable since the absorber is to shields, absorbs, or reduces the number of photons going into the detector, then the more increase its thickness the more it does this work and the lesser the photons that get to the detector and then the lower the number of counted photons. The exponential relationship for photon absorption suggests that, theoretically, complete absorption of the beam of photon radiation never really occurs, but in a practical sense, exponential attenuation and absorption can be used to reduce most of the beam intensities to imperceptible levels.

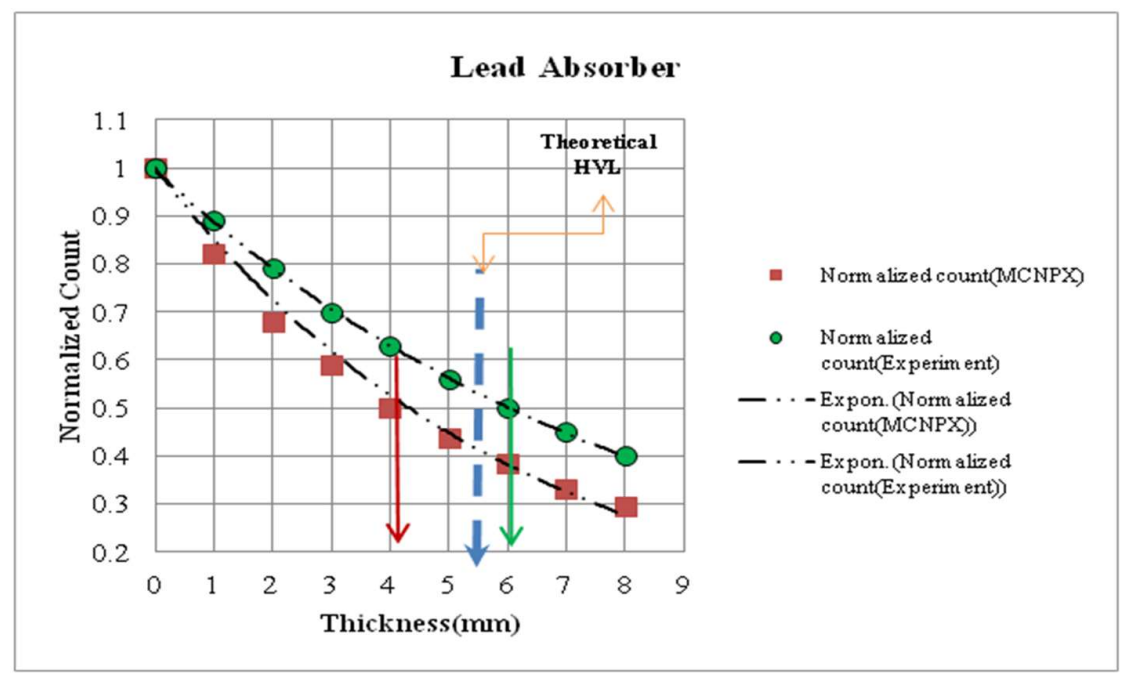

Figure 9: MCNPX, Experimental and Theoretical Results for Pb Absorber

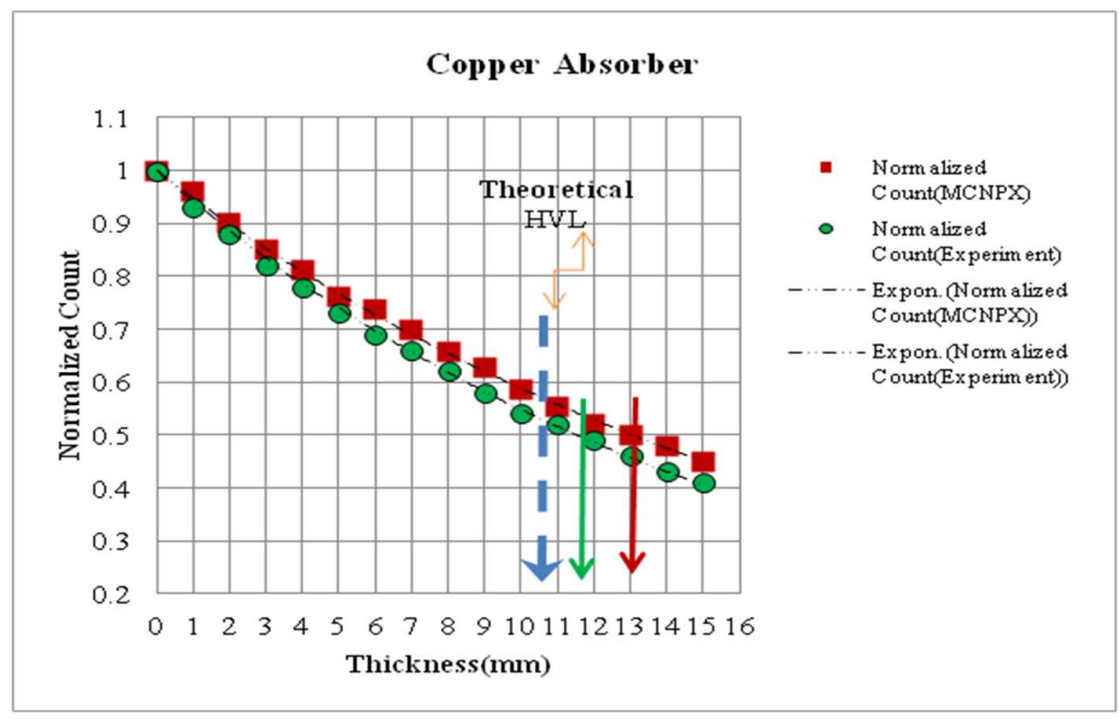

Figure 10: MCNPX, Experimental and Theoretical Results for $\mathrm{Cu}$ Absorber 


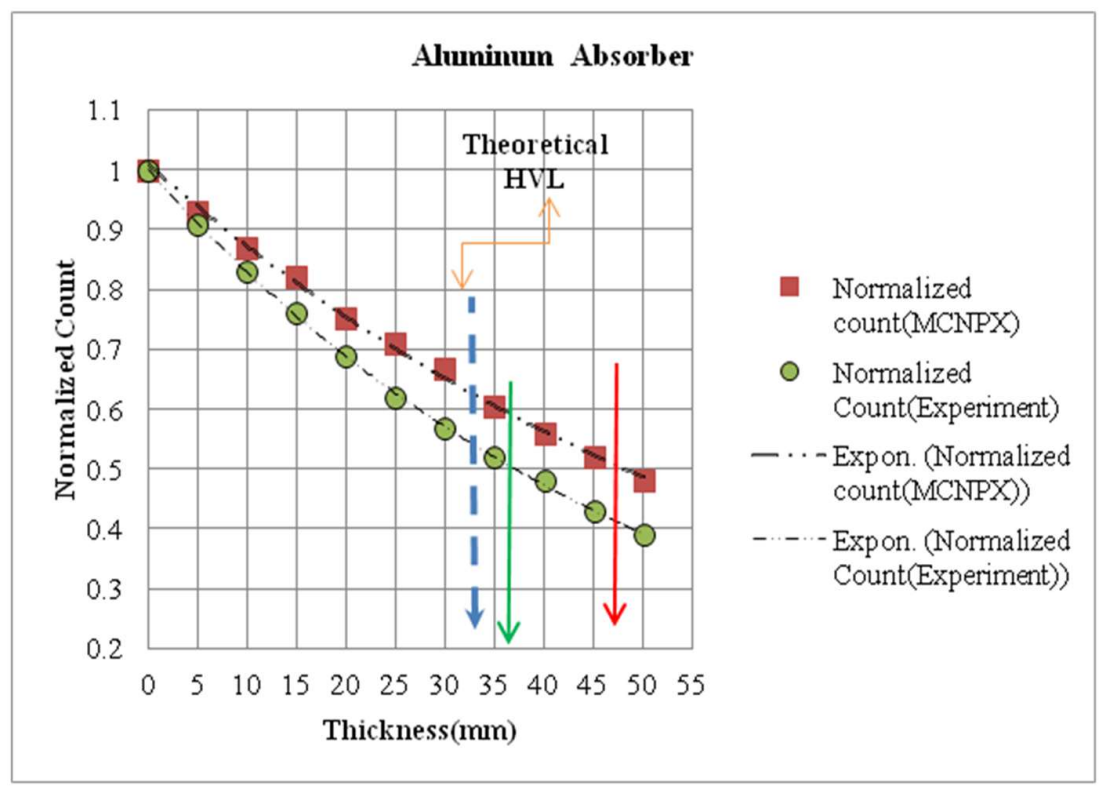

Figure 11: MCNPX, Experimental and Theoretical Results for Al Absorber

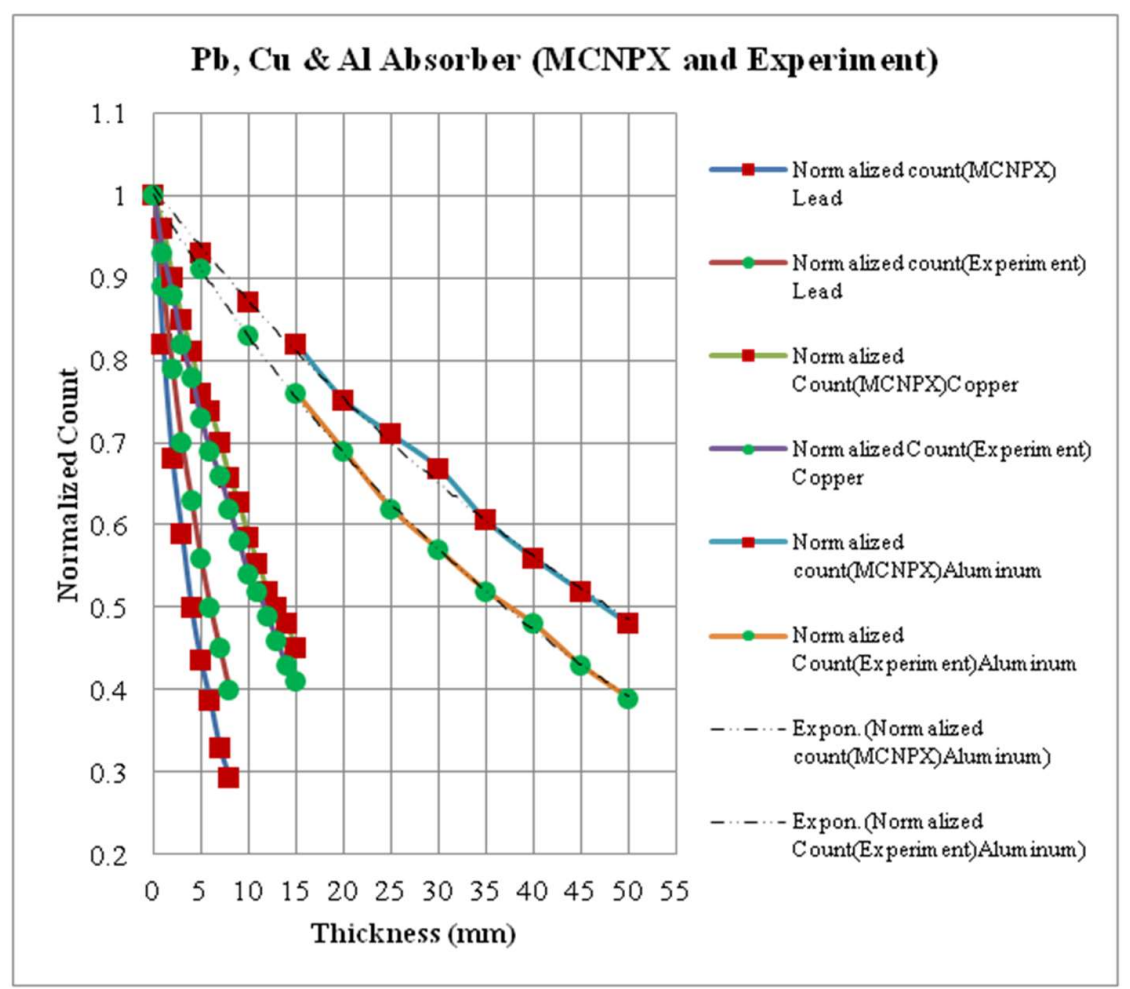

Figure 12: MCNPX and Experimental Results for three absorbers ( $\mathrm{Pb}, \mathrm{Cu}, \mathrm{Al})$ absorber

It has been found from Figure 12 that the experiment result represents a narrower thickness of absorber than MCNPX results to accomplish an intensity reduction of the incident radiation by a factor of 2 and almost comparable HVL values with the MCNPX. Even though the MCNPX results were higher (in the case of Cu and $\mathrm{Al})$ than the experimental esteems, they show a similar exponential variety in relative intensity with thickness, same concerning Lead, where MCNPX results are lower than the experimental outcome. That is, for the Lead case, a $0.8 \mathrm{~cm}$ thick absorber fulfilled a $60 \%$ reduction of the incidence of radiation in the experiment. A similar thickness fulfilled a $70 \%$ decrease in MCNPX. For the Copper case, $1.5 \mathrm{~cm}$ fulfilled a $59 \%$ intensity drop in 
the experiment, while in MCNPX fulfilled a $55 \%$ reduction. For Aluminum, $5.0 \mathrm{~cm}$ fulfilled a $61 \%$ intensity decrease in the experiment, while in MCNPX fulfilled a $52 \%$ decrease. A study by Ibrahim A. Alrammah, 2016, mentioned that this may be because of several reasons- a) in the experiment, various layers (sheets) of absorber were utilized. While in the simulation they were considered as one layer; $b$ ) the noise present in the experiment from encompassing objects that can be treated as an absorber, while it isn't the situation in the simulation; c) the qualities of the material (for example temperature, pressure, geometry, thickness) applied in the experiment may differ slightly from that of the simulation; Another reason for the slight discrepancy of the experimental and simulation results may be for the unknown detector efficiency, detector calibration factor and experimental geometry efficiency result in overestimating the experimental result. Furthermore, the isotopic composition of Lead, Copper, and Aluminum were not precisely defined in the MCNPX code.

\section{CONCLUSION}

Through $48.86 \mathrm{mCi} 662 \mathrm{keV}$ Caesium-137 gamma-ray source shielding experiment and simulation of it with MCNPX code were done in three shielding materials Lead, Copper, and Aluminum. From the simulation results, the gamma photon transport in three shielding materials was adequately modeled using MCNPX code and an elective technique for the experimental method, because of their flexibility and convenience in defining geometry. One reason for the slight variations between MCNPX and Experimental outcomes as mentioned in the previous section might be the isotopic composition of Lead, Copper, and Aluminum shielding materials which were not all around characterized in MCNPX code due to the unavailability of materials data to approach the exact value. This study can be extremely valuable for wide uses of materials for gamma-rays shielding and utilization of standardized geometry of Monte Carlo simulation for radiation physics, shielding, and radiation protection. This standard geometry can be used for studying the shielding effects in different shielding materials.

\section{ACKNOWLEDGMENT}

We desire to acknowledge the staff and personnel of the Malaysian Nuclear Agency for necessary equipment, materials, and all-around support in this work. We wish also to concede IAEA for their funding.

\section{REFERENCES}

Boston Andrew, 2000. Introduction to MCNP-the Monte Carlo transport code, Olive Lodge Laboratory, pp 240. Denise B. Pelowitz, editor, 2011, User's Manual, MCNPX ${ }^{\mathrm{TM}}$, Version 2.7.0.

Etherington, H, 1958. Nuclear Engineering Handbook, McGraw -Hill book company, pp 7-61: 7-111.

Ibrahim A. Alrammah, 2016. Application of MCNP Code in Shielding Design for Radioactive Sources, International Journal of Chemical, Environmental \& Biological Sciences 4(1).

Judith F. Briesmeister, 2000. MCNP -A General Monte Carlo N-Particle Transport Code: Version 4C, USA: Los Alamos Science, pp. 4-36.

McConn R. J. Jr, Gesh C.J, Pagh R.T, Rucker R.A, Williams R.G III, 2011. Compendium of Material Composition Data for Radiation Transport Modeling, Rev. 1, USA: Pacific Northwest National Laboratory.

Metropolis N., 1987. The Beginning of the Monte Carlo method, USA: Los Alamos Science.

X-5 Monte Carlo Team, 2005. User's Guide, MCNP- A General Monte Carlo N-Particle Transport Code, Version 5, Volume I.

(C) 2021 the Authors. Journal of Engineering Science published by Faculty of Civil Engineering, Khulna University of Engineering \& Technology. This is an open access article under the terms of the Creative Commons AttributionNonCommercial-NoDerivatives License, which permits use and distribution in any medium, provided the original work is properly cited, the use is non-commercial and no Modifications or adaptations are made. 